\title{
Soporte social percibido por las personas con enfermedad crónica y sus cuidadores familiares en cinco macro regiones geográficas de Colombia
}

\author{
Social support perceived in people with chronic disease and their family caregivers in five macro regions of \\ Colombia
}

Lucy Barrera-Ortiz1, María Stella Campos², Karina Gallardo-Solarte3, Rosa del Carmen Coral-lbarra4,

Adriana Hernández-Bustos 5

1 Enfermera. Magíster en Enfermería. Profesora Titular (p) Universidad Nacional de Colombia. Bogotá, Colombia. e-mail: Ibarrerao@unal.edu.co

2 Enfermera, Magíster en Enfermería en Salud Familiar. Docente. Programa de Enfermería Universidad de Santander (UDES). Bucaramanga, Colombia. e-mail: scamposaldana@hotmail.com

3 Enfermera. Magíster en Enfermería. Docente Investigadora Universidad Mariana. Pasto, Colombia. e-mail: karinagallardo@umariana.edu.co

4 Magister en Docencia Universitaria. Docente Facultad de Enfermería. Universidad de Ciencias Aplicadas y Ambientales U.D.C.A. Bogotá, Colombia. e-mail: rcoral@udca.edu.co

5 Enfermera. Magíster en Tecnologías de la información aplicadas a la educación. Docente Universidad de Cundinamarca. Fusagasugá, Colombia. e-mail: adrianaher7714@hotmail.com enfermedad crónica y sus cuidadores familiares en cinco macro regiones geográficas de Colombia. Univ. Salud. 2016;18(1):102-11. DOI: http://dx.doi.org/10.22267/rus.161801.23

\section{Resumen}

Objetivo: Describir y comparar el soporte social percibido por las personas con enfermedad crónica y sus cuidadores familiares. Materiales y métodos: Estudio de tipo descriptivo, comparativo, de corte transversal desarrollado con una muestra de 1.257 personas con enfermedad crónica y 1.184 cuidadores familiares en las cinco macro regiones geográficas de Colombia entre los años 2012 - 2014. Resultados: Las condiciones de las personas con enfermedad crónica y de los cuidadores familiares participantes en el estudio, reflejan predominio femenino con niveles de escolaridad medio-bajo, y con residencia en la zona urbana. La mitad de los participantes tienen pareja estable y trabajo remunerado. La situación de enfermedad crónica se presenta para la mayoría, por más de un año. La familia es la principal fuente de apoyo social y a pesar de ello, la cuarta parte de personas con enfermedad crónica perciben que son una carga para sus familias y la tercera parte de los cuidadores familiares, percibe sobrecarga intensa con el cuidado. Se reporta un nivel adecuado de soporte social, en especial en las regiones Amazonía y Orinoquía. Conclusiones: Las personas con enfermedad crónica y sus cuidadores familiares tienen niveles de soporte social percibido heterogéneos en las diferentes macro regiones geográficas de Colombia. El soporte social percibido es insuficiente para respaldar la experiencia del cuidado de la salud de estas personas.

Palabras clave: Apoyo social; enfermedad crónica; Colombia. (Fuente: DeCS, Bireme). 


\begin{abstract}
Objective: To describe and compare the social support perceived by people with chronic disease (CD) and their family caregivers (FC) in Colombia. Materials and methods: This is a descriptive, comparative cross-sectional study developed with a sample of 1257 people with CD and 1184 FC in the five macro geographical regions of Colombia between 2012 and 2014. Results: The conditions of participants with CD and their FC reflects female predominance with medium-low levels of schooling who live in the urban area. Half of the participants have a regular partner as well as a paid job. The situation of chronic disease occurs mostly for over a year. The family is the main source of social support and yet a quarter of people with CD perceive to be a burden to their families and the third of the FC perceived intense care overload. An adequate level of social support is reported, especially in the Amazon and Orinoco regions. Conclusions: People with chronic illness and their family caregivers have heterogeneous levels of perceived social support in the different macro geographical regions of Colombia. The perceived social support is insufficient to support the experience of health care of these people.
\end{abstract}

Keywords: Social support; chronic disease; Colombia. (Source: DeCS, Bireme).

\section{Introducción}

Las enfermedades crónicas desde hace varios años encabezan la lista de los índices de morbilidad y mortalidad a nivel mundial.1 Respecto a esto, gobiernos y organizaciones en diferentes países alrededor del mundo han dirigido recursos económicos y de talento humano en la lucha por disminuir el impacto de estas enfermedades a nivel personal, social, económico y cultural.2

Debido a la naturaleza de la enfermedad crónica (EC), las personas que la padecen y sus cuidadores familiares (CF) enfrentan cuatro tipos de problemas asociados con el cuidado: los que surgen de la necesidad de modificar sus estilos de vida para mejorar el pronóstico; los que implican el manejo del dolor la limitación y la mutilación asociada con la EC; la alteración de la socialización; y el enfrentamiento de una gran pérdida o la muerte.

Aunque la experiencia de la EC en cada individuo es distinta y sobre ella influyen diversas variables, el soporte social existente facilita el afrontamiento, la adaptación y disminuye las consecuencias psicológicas y psicosociales de la enfermedad.3 Este apoyo social hace referencia a los comportamientos de solidad provenientes de diferentes personas, sean estas pertenecientes al sistema de salud, familiares, vecinos, amigos o cualquier otra persona que esté en condiciones de brindar ayuda.4
El soporte social descrito también como apoyo social percibido, se considera un elemento esencial en la interacción de los seres humanos, es la base para afrontar situaciones que afecten el desarrollo, desempeño y realización de las personas; sus referentes se encuentran registrados en la literatura, a partir de la década del setenta y cobra especial relevancia en los últimos años, como factor determinante $\mathrm{e}$ influyente en los procesos salud-enfermedad.5

El apoyo social se define como "la diversidad de comportamientos naturales de ayuda de los que los individuos son receptores en interacciones sociales, la ayuda tangible, interacción intima, la guía, la retroalimentación y la integración social positiva" ${ }_{5,6}$

Las funciones del apoyo social pueden ser requeridas dentro de la experiencia de la enfermedad crónica por el paciente y por su cuidador y se pueden clasificar en cuatro grupos complementarios entre sí: 1) el emocional que provee al individuo sentimientos de estima, afecto, confianza, seguridad, 2) el valorativo, representa la sensación percibida por el individuo en la que puede contar con alguien, y sentirse acompañado socialmente, 3) el informacional, también conocido como consejo o guía cognitiva, que ayuda a definir y enfrentar los eventos problemáticos mediante asesoramiento por parte de la red y 4) el instrumental constituye la ayuda tangible y material en la solución a un problema.6,7 
En situaciones de enfermedad crónica, el soporte o apoyo social representa el apoyo para que el paciente y el cuidador familiar afronten con facilidad la experiencia de cuidar. $6,8,9 \mathrm{Al}$ respecto, varias investigaciones sugieren que los niveles de soporte social se asocian en forma directa con los resultados en las personas con EC, de manera que la falta de soporte se asocia con elevada mortalidad.10-12

Más aún, estudios realizados, en población con enfermedad crónica, muestran el impacto positivo del soporte social con relación al desarrollo de mecanismos de afrontamiento, control de estrés, disminución de la ansiedad, depresión y aislamiento generados de la experiencia de cronicidad. 13

Si bien algunas de las investigaciones citadas se han adelantado en este el contexto colombiano, dicho contexto es diverso en sus aspectos socioculturales y económicos, por lo cual hacer una aproximación al soporte social percibido en las diferentes regiones de Colombia, adquiere especial interés, con el propósito de establecer un diagnóstico situacional del soporte social en la EC, buscando que el mismo sirva para generar intervenciones que atiendan las necesidades especiales de las personas con EC y sus familias.

Este estudio buscó describir el soporte social percibido, tanto por las personas con enfermedad crónica, como por sus cuidadores familiares participantes de las cinco regiones de Colombia, durante el año 2012 - 2014, con el fin de contar con un diagnóstico línea de base para evaluar el efecto de intervenciones que fortalezcan el soporte social percibido, en una fase posterior.

\section{Materiales y métodos}

El presente estudio fue aprobado por el Comité de Ética Institucional, previa justificación y revisión de cumplimiento de los aspectos éticos considerados en la normativa vigente.14 Los autores acogieron la política ambiental para el manejo de los recursos, en especial el uso de papel. $_{.15} \mathrm{Su}$ abordaje fue de tipo descriptivo y de corte transversal, realizado entre los años 2012 y
2014. La muestra a conveniencia estuvo conformada por 1.257 personas con enfermedad crónica y 1.184 cuidadores familiares de las cinco macro regiones geográficas de Colombia, captados desde los programas docenteasistenciales, en los centros de atención primaria, hospitales, grupos o asociaciones de pacientes a los que asistían en sus comunidades. Su distribución por regiones del país, como se ilustra en la tabla 1.

Dentro de los criterios de inclusión para las personas con EC se contemplaron: (a) ser mayor de 18 años, (b) tener diagnóstico confirmado de EC, (c) tener la capacidad de responder las preguntas de los instrumentos. Para los cuidadores familiares: (a) ser cuidador familiar principal de persona con EC con quien se tenga vínculo de parentesco o cercanía, (c) estar en la capacidad de responder las preguntas de los instrumentos. Dentro de los criterios de exclusión en personas con EC y cuidadores familiares se tuvieron en cuenta: (a) estar bajo el consumo de sustancias psicoactivas al momento de diligenciar el instrumento, (b) no firma del consentimiento informado.

Las personas con EC y los cuidadores familiares que cumplían con los criterios de inclusión fueron invitados para participar en el estudio, por auxiliares de investigación. A estos se les explicó el objetivo del estudio, el manejo de la información y la confidencialidad de la misma y su derecho a cancelar su participación en él cuando lo desearan. Quienes aceptaron participar en el estudio firmaron el consentimiento informado y conservaron una copia del mismo. Una vez recibido el consentimiento informado, los participantes acordaron con los auxiliares de investigación el lugar y la fecha de mayor conveniencia para diligenciar los instrumentos, (hogar de los participantes, centro de atención de salud u hospital). Los formatos fueron autoadministrados en los casos en que la persona con enfermedad crónica y el cuidador familiar podían responderlos por sí solos. Para las personas analfabetas, los instrumentos fueron administrados por el auxiliar de investigación, quien se encargó de hacer las preguntas $y$ diligenciar el instrumento. 
Tabla 1. Participantes en el diagnóstico de soporte social percibido: Cinco macro regiones geográficas de Colombia. 2012-2014

\begin{tabular}{|c|c|c|c|c|}
\hline $\begin{array}{l}\text { Región de } \\
\text { Colombia }\end{array}$ & Programas docente asistencial & Sitio de residencia & $\begin{array}{c}\mathrm{N}^{\circ} \text { de } \\
\text { pacientes }\end{array}$ & $\mathrm{N}^{\circ}$ de cuidadores \\
\hline Amazonía & Universidad Nacional de Colombia & $\begin{array}{l}\text { Leticia } \\
\text { Puerto Asís }\end{array}$ & 201 & 201 \\
\hline Andina & $\begin{array}{l}\text { Universidad de Ciencias Aplicadas y Ambientales UDCA } \\
\text { Universidad de Cundinamarca } \\
\text { Universidad Mariana de Pasto } \\
\text { Universidad de Santander UDES } \\
\text { Universidad Nacional de Colombia } \\
\text { Universidad de Cundinamarca }\end{array}$ & $\begin{array}{l}\text { Bogotá } \\
\text { Girardot } \\
\text { Chía } \\
\text { Cajicá } \\
\text { Tocancipá } \\
\text { Pasto } \\
\text { Bucaramanga }\end{array}$ & 767 & 745 \\
\hline Caribe & Universidad Nacional de Colombia & Valledupar & 96 & 96 \\
\hline Orinoquía & Universidad Nacional de Colombia & $\begin{array}{l}\text { Arauca } \\
\text { Granada Meta } \\
\text { Villavicencio }\end{array}$ & 56 & 52 \\
\hline Pacífico & $\begin{array}{l}\text { Universidad Mariana de Pasto } \\
\text { Universidad Nacional de Colombia }\end{array}$ & $\begin{array}{l}\text { Buenaventura } \\
\text { Tumaco }\end{array}$ & 137 & 90 \\
\hline Total & & & 1.257 & 1.184 \\
\hline
\end{tabular}

Para describir algunas características de los participantes y su percepción de soporte social se emplearon dos instrumentos: el primero, la encuesta de caracterización para el cuidado de la diada - conocida como GCPC-UN-D, que cuenta con las variables necesarias para la caracterización de una diada persona con enfermedad crónica - cuidador familiar que contiene 42 ítems y tres dimensiones: 1) Las condiciones y perfil socio demográfico de la diada. 2) La percepción de carga y apoyo. 3) Los medios de información y comunicación.

El segundo, fue el cuestionario para la valoración del soporte social MOS: puede ser aplicado a personas con EC y a sus cuidadores familiares, utilizando la versión respectiva. Este cuestionario desarrollado por Sherbourne y Stewart en 1991,16 mide el constructo apoyo social, específicamente el apoyo funcional que se refiere a la percepción de apoyo. El apoyo funcional es multidimensional, comprende lo emocional (amor, empatía), instrumental (tangible), informativo (consejos y feedback), valoración (ayuda a la autovaloración) y compañerismo durante el tiempo libre. Cuenta con 20 ítems, de los cuales el primero se relaciona con el tamaño de la red social y los restantes se refieren a las dimensiones del apoyo social: emocional, instrumental, interacción social positiva y apoyo afectivo.
La distribución factorial de los ítems es: apoyo emocional (ítems 3,4,8,9,13,16,17 y 19), apoyo instrumental (ítems $2,5,12,15$ ), interacción social positiva (ítems $7,11,14,18$ ) y apoyo afectivo (ítems 6,10,20). Se mide con una escala de 5 puntos en donde a mayor sea el puntaje, mayor es el apoyo social percibido. Este cuestionario ha sido analizado psicométricamente en Latinoamérica. 17 En Colombia, este cuestionario fue validado por Londoño et al., su consistencia interna tuvo un alfa de Cronbach de 0.941 total y para los componentes se reportó entre 0.921 y 0.736 , lo que corroboró la consistencia de la prueba.18

Se utilizó estadística descriptiva con presentación de frecuencias absolutas y relativas para la caracterización de la diada del estudio. (Personas con enfermedad crónica, los cuidadores familiares de personas con enfermedad crónica). Respecto al soporte social percibido en personas con EC y CF, se aplicó estadística descriptiva utilizando medidas de tendencia central y de dispersión para la totalidad del instrumento y las dimensiones. Adicionalmente, el soporte social se analizó a partir de los niveles de estratificación propuestos: alto medio y bajo.

La estratificación de estos rangos fue resultado del análisis estadístico y la aplicación de la regla 
de Dalheinius. En la tabla 2, se detallan los rangos de puntuación correspondientes a personas con EC y CF.

Tabla 2. Valores clasificación de soporte social MOSpersonas con enfermedad crónica y cuidadores familiares de personas con enfermedad crónica

\begin{tabular}{llrr}
\hline $\begin{array}{c}\text { Soporte y } \\
\text { dimensiones }\end{array}$ & Clasificación & $\begin{array}{c}\text { Persona } \\
\text { con EF }\end{array}$ & $\begin{array}{c}\text { CF de } \\
\text { persona } \\
\text { con EC }\end{array}$ \\
\hline Soporte & Bajo & 19 a 62 & $19-60$ \\
social & Medio & $63-81$ & $61-79$ \\
& Alto & $82-95$ & $90-95$ \\
Dimensión: & Bajo & $8-24$ & $8-24$ \\
Apoyo & Medio & $25-33$ & $25-33$ \\
emocional & Alto & $34-40$ & $34-40$ \\
& & & \\
Dimensión: & Bajo & $4-12$ & $4-11$ \\
Apoyo & Medio & $13-17$ & $12-16$ \\
instrumental & Alto & $18-20$ & $17-20$ \\
& & $4-12$ & $4-12$ \\
Dimensión: & Bajo & $13-16$ & $13-16$ \\
Interacción & Medio & $17-20$ & $17-20$ \\
social & Alto & & \\
positiva & & & \\
& & $3-9$ & $3-9$ \\
Dimensión: & Bajo & $10-12$ & $10-12$ \\
Apoyo & Medio & $13-15$ & $13-15$ \\
afectivo & Alto & & \\
\hline
\end{tabular}

Con el fin de comparar cada una de las dimensiones, atendiendo a que no cuentan con el mismo número de ítems, se realizó una estandarización de las variables en porcentaje de 0 a 100, a partir de los puntajes obtenidos por cada participante.

Para la comparación de las regiones se aplicó la prueba no paramétrica de Kruskal-Wallis y la prueba chi cuadrado para variables sociodemográficas, tanto de los pacientes, como de los cuidadores.

\section{Resultados}

\section{Características sociodemográficas de la persona con enfermedad crónica}

El $60,5 \%$ de personas con EC son mujeres. En cuanto a la edad el $20,6 \%$ tiene 35 años o menos, el $32,6 \%$ de 36 a 59 años y el $46,8 \% 60$ o más. Respecto al nivel educativo, el $69 \%$ tienen menos de un bachillerato completo. El $85,9 \%$ proceden de la zona urbana y el $84,6 \%$ de las personas con
EC mantienen una unión de pareja estable. El $46,9 \%$ tiene una actividad económica remunerada, bien sea por empleo o trabajo independiente.

En cuanto al compromiso religioso el 30,1\% de los pacientes tiene un nivel en medio o alto, con una afinidad religiosa católica en el 78\% de los casos.

Con respecto a la funcionalidad, el $34 \%$ de las personas con EC tienen algún nivel de dependencia. De igual forma, el 21\% tiene alteración cognitiva y de ellos el 5,9\% la presenta moderada y el 2,3\% severa.

En cuanto a las patologías el 29,10\% presenta trastornos cardiovasculares, el 15,10\% cáncer, el $13,4 \%$ enfermedad neurológica, el 9,4\% enfermedad del aparato locomotor y el 4,10\% enfermedad renal; con una duración de la enfermedad de más de 19 meses en el 67,4\% de casos. El 45,7\% reconoce tener más de un cuidador, mientras que el 50,4\% manifiesta requerir apoyo de un CF seis horas o menos al día y el 21,3 lo requiere durante 24 horas. Entre los apoyos percibidos por las personas con EC se destaca el psicológico en $42 \%$ sin embargo, el $59,9 \%$ expresan no estar satisfechos con el apoyo que reciben. Con respecto al apoyo familiar el $90,4 \%$ señalan percibirlo y sólo el $15,5 \%$ no se encuentran satisfechos con este.

El 55,8\% perciben apoyo religioso y de ellos el $34,7 \%$, expresan que el mismo no les satisface. Con respecto al apoyo económico el $62,1 \%$ de personas con EC lo percibe y de ellos el 39,3\% señala que este apoyo les satisface. Frente al apoyo social el 58,8\% de las personas lo percibe y expresan satisfacción con el mismo el 42,1\%.

El 20,8 \% de las personas con EC siente que es una carga alta o muy alta para su familia y en cuanto al bienestar el 62,2\% percibe bienestar físico, el $73,50 \%$ psicológico, el $75,8 \%$ social y el $85,9 \%$ espiritual.

La valoración del nivel de apropiación de las TIC refleja que si bien las mismas son útiles, distan de ser vistas como un elemento fundamental para 
facilitar o apoyar el cuidado de la salud en las situaciones de EC.

El 39,6\% de las personas con EC usa TIC para apoyar su cuidado y el $64,1 \%$ percibe bajo apoyo para su cuidado con el empleo de TIC's. Cuando califican las TIC según la prioridad frente a la utilidad percibida para recibir información en el cuidado, el $66,6 \%$ señala una alta prioridad para la TV, para la radio el $49.9 \%$ y para el computador el 23,8\% de los casos. Para el teléfono el 32,6\% y para el internet el $25 \%$ de ellos.

\section{Características sociodemográficas del cuidador familiar}

El 74,7\% de los CF son mujeres. En cuanto a la edad el 39,3\% tiene menos de 35 años, el 43,7\% de 36 a 59 años y el 16,9\% tiene 60 o más años. Respecto al nivel educativo el 56,6\% tienen menos de un bachillerato completo. El 74,4\% proceden de la zona urbana y solo el $59,8 \%$ cuenta con unión de pareja estable. El 46,9\% tiene una actividad económica remunerada, bien sea por empleo o trabajo independiente.

En cuanto al compromiso religioso el $77 \%$ de los cuidadores tienen un nivel medio o alto y una filiación religiosa católica en el 77,8\% de los casos, seguido por la cristiana.

Con respecto a la funcionalidad llama la atención que el 8,6\% de los CF señala tener algún nivel de dependencia y el $5.4 \%$ presentan alteración cognitiva.

El 39,7\% de los CF reporta tener alguna enfermedad, siendo la diabetes la más común, con $29,6 \%$ de personas que la reportan. El 72\% de los cuidadores reportan cuidar a su familiar durante el tiempo que llevan con la enfermedad y el $44,3 \%$ manifiesta no ser el único cuidador de su familiar. El 48,8\% reporta proporcionar cuidado por lo menos de 6 horas al día, mientras el 29,6\% señala hacerlo las 24 horas.

Con respecto a los apoyos percibidos se destaca el psicológico en un 47,9\%, sin embargo, el 40,5\% no están satisfechos con el apoyo que reciben. Con respecto al apoyo familiar el $83,2 \%$ señalan percibirlo y $14,6 \%$ manifiestan no estar satisfechos con éste.

En cuanto al apoyo religioso el $60,3 \%$ de los CF lo perciben y de ellos el 44,9\% expresan que el mismo no les satisface. Con respecto al apoyo económico el 64,6\% lo percibe y de ellos el 57,2\%, señala que este apoyo les satisface. Frente al apoyo social el $60,6 \%$ de los CF lo percibe y expresan satisfacción con el mismo el 20,5\%.

El 20,8 \% de los CF sienten que tienen sobrecarga ligera con el cuidado en el 38,9\% de casos e intensa en el $22,2 \%$. En cuanto al bienestar, $85,5 \%$ de los casos percibe bienestar físico, el $85,3 \%$ psicológico, el $87,9 \%$ social y el $90 \%$ espiritual.

La valoración del nivel de apropiación de las TIC refleja que si bien las mismas son útiles, distan de ser vistas como un elemento fundamental para facilitar o apoyar el cuidado de la salud en las situaciones de EC.

El 52,1\% de los CF usa TIC para apoyar su cuidado y el $51,9 \%$ perciben bajo apoyo para su cuidado con el empleo de éstas herramientas. Cuando califican las TIC según la prioridad frente a la utilidad percibida para recibir información en el cuidado, se observa una priorización de la TV en el $64,8 \%$ de casos, la radio en el $44,7 \%$, el computador en el $35,2 \%$, el teléfono en el 39,3\% y el internet en el 40,9\%.

\section{Percepción de soporte social de las personas con EC}

Se identificó un promedio de 6 amigos y parientes con los que cuentan los pacientes. Con porcentajes posibles de 0 a 100, los resultados del soporte social percibido en personas con enfermedad crónica, participantes del estudio a nivel nacional, indican una media en el soporte general de 62,7\%. Respecto a las dimensiones, apoyo emocional se encontró un promedio de $76,3 \%$, interacción social positiva 78,01\%, apoyo afectivo $80,9 \%$ y apoyo instrumental $81,3 \%$. (Tabla 3). 
Tabla 3. Soporte social percibido en personas con enfermedad crónica por niveles y regiones de Colombia

\begin{tabular}{rlrrrrr}
\hline Región & Estadístico & $\begin{array}{c}\text { Soporte } \\
\text { Social total }\end{array}$ & $\begin{array}{c}\text { Apoyo } \\
\text { emocional }\end{array}$ & $\begin{array}{c}\text { Interacción } \\
\text { social }\end{array}$ & $\begin{array}{c}\text { Apoyo } \\
\text { instrumental }\end{array}$ & \multicolumn{1}{c}{$\begin{array}{c}\text { Apoyo } \\
\text { afectivo }\end{array}$} \\
\hline Amazonía & Media & 70,1 & 87,4 & 87,4 & 87,8 & 87,9 \\
& Desviación & 20,9 & 26,1 & 26,5 & 26,4 & 26,5 \\
& estándar (DS) & & & & 0,0 & 0,0 \\
& Mínimo & 0,0 & 0,0 & 10,0 & 100,0 & 100,0 \\
& Máximo & 80,0 & 100,0 & 70,8 & 80,3 \\
Andina & Media & 61,5 & 73,7 & 21,3 & 24,3 \\
& DS & 17,1 & 24,1 & 24,0 & 0,0 & 0,0 \\
& Mínimo & 0,0 & 0,0 & 0,0 & 100,0 & 100,0 \\
& Máximo & 80,0 & 100,0 & 100,0 & 77,3 & 76,8 \\
& Media & 60,7 & 75,5 & 74,6 & 20,4 & 20,7 \\
& DS & 13,4 & 16,9 & 18,9 & 0,0 & 0,0 \\
& Mínimo & 7,4 & 12,5 & 12,5 & 100,0 & 100,0 \\
& Máximo & 80,0 & 100,0 & 100,0 & 88,2 & 86,9 \\
& Media & 67,6 & 81,6 & 84,7 & 17,7 & 19,1 \\
& DS & 14,7 & 20,0 & 19,4 & 25,0 & 25,0 \\
& Mínimo & 19,0 & 18,8 & 25,0 & 100,0 & 100,0 \\
& Máximo & 80,0 & 100,0 & 100,0 & 74,8 & 74,8 \\
& Media & 58,9 & 73,3 & 72,2 & 23,1 & 26,7 \\
& DS & 18,8 & 24,5 & 25,8 & 12,5 & 0,0 \\
& Mínimo & 9,5 & 12,5 & 0,0 & 100,0 & 100,0 \\
\hline
\end{tabular}

Se identifica que las personas con EC de las regiones Orinoquía y Amazonía reportan los mayores niveles de soporte social percibido, en contraste con las de las regiones Andina, Caribe y Pacífico.

Al realizar la comparación de las regiones utilizando la prueba no paramétrica de Kruskal Wallis para muestras independientes, se confirma que el soporte social percibido de personas con EC en general y con cada una de sus dimensiones, es diferente en cada una las regiones geográficas de Colombia $(\mathrm{p}<0.05)$

Por otro lado, al realizar el análisis con la prueba chi cuadrado, se identifica que la región influye en la categorización soporte social percibido de personas con EC en general y con cada una de sus dimensiones.

\section{Percepción de soporte social de los CF}

En lo referente a la percepción de soporte social de los CF se identificó un promedio 6,48 personas conocidas de quienes perciben apoyo, con un mínimo de cero (o) y máximo de 30 en algunos casos, con una desviación estándar de 5,88.
Con porcentajes posibles de 0 a 100, los resultados del soporte social percibido en cuidadores familiares de personas con enfermedad crónica, participantes del estudio en las regiones, indican una media en el soporte social de $75,2 \%$. Respecto a las dimensiones, apoyo emocional presentaron un promedio de $74,2 \%$, apoyo instrumental $71,9 \%$, interacción social positiva $77,1 \%$, y apoyo afectivo $80,1 \%$, siendo el más alto. (Tabla 4).

Se identificó que los CF de las regiones Orinoquía y Amazonía reportan los mayores niveles de soporte social percibido, mientras los cuidadores de las regiones Andina, Caribe y Pacífico, niveles más bajos. Al realizar la comparación de las regiones utilizando la prueba no paramétrica de Kruskal Wallis para muestras independientes se confirma que el soporte social percibido de CF de personas con EC en general y con cada una de sus dimensiones es diferente en cada una las regiones geográficas de Colombia $(\mathrm{p}<0.05)$.

Por otro lado, al realizar el análisis con la prueba chi cuadrado, se identifica que la región influye en la categorización soporte social percibido de cuidadores familiares de personas con enfermedad crónica, en general y con cada una de sus dimensiones. 
Tabla 4. Soporte social percibido en cuidadores familiares de personas con enfermedad crónica por regiones de Colombia

\begin{tabular}{|c|c|c|c|c|c|c|}
\hline Dimensión & Estadístico & Andina & Amazonía & Pacífico & Caribe & Orinoquía \\
\hline \multirow[t]{4}{*}{ Soporte social } & Media & 71,5 & 87,5 & 73,2 & 74,1 & 88,2 \\
\hline & $\begin{array}{l}\text { Desviación } \\
\text { estándar (DS) }\end{array}$ & 22,3 & 28,0 & 20,6 & 16,7 & 13,1 \\
\hline & Mínimo & 0,0 & 0,0 & 17,1 & 34,2 & 56,6 \\
\hline & Máximo & 100,0 & 100,0 & 100,0 & 100,0 & 100,0 \\
\hline \multirow{4}{*}{$\begin{array}{c}\text { Apoyo } \\
\text { emocional }\end{array}$} & Media & 70,1 & 87,4 & 71,6 & 74,7 & 88,3 \\
\hline & DS & 24,7 & 28,2 & 22,9 & 17,5 & 13,4 \\
\hline & Mínimo & 0,0 & 0,0 & 15,6 & 34,4 & 59,4 \\
\hline & Máximo & 100,0 & 100,0 & 100,0 & 100,0 & 100,0 \\
\hline \multirow{4}{*}{$\begin{array}{c}\text { Apoyo } \\
\text { instrumental }\end{array}$} & Media & 67,2 & 87,4 & 69,7 & 69,8 & 86,5 \\
\hline & DS & 26,8 & 27,7 & 24,0 & 19,7 & 17,3 \\
\hline & Mínimo & 0,0 & 0,0 & 6,3 & 25,0 & 18,8 \\
\hline & Máximo & 100,0 & 100,0 & 100,0 & 100,0 & 100,0 \\
\hline \multirow{4}{*}{$\begin{array}{l}\text { Interacción } \\
\text { social }\end{array}$} & Media & 73,9 & 87,5 & 75,7 & 75,1 & 88,8 \\
\hline & DS & 23,5 & 798,2 & 523,6 & 294,8 & 204,3 \\
\hline & Mínimo & 0,0 & 28,3 & 22,9 & 17,2 & 14,3 \\
\hline & Máximo & 100,0 & 0,0 & 12,5 & 25,0 & 37,5 \\
\hline \multirow[t]{4}{*}{ Apoyo afectivo } & Media & 77,9 & 87,9 & 78,9 & 77,2 & 89,3 \\
\hline & DS & 23,9 & 28,2 & 21,8 & 20,0 & 14,4 \\
\hline & Mínimo & 0,0 & 0,0 & 8,3 & 25,0 & 50,0 \\
\hline & Máximo & 100,0 & 100,0 & 100,0 & 100,0 & 100,0 \\
\hline
\end{tabular}

\section{Discusión}

Al revisar las características de los participantes se evidencia una preponderancia del género femenino con un 60,5 \% de personas con EC y $74,4 \%$ de cuidadores familiares, con predominancia de edad media en el $32,6 \%$ de los casos y un poco mayor en el caso de las personas con EC con 46,8\% que sobrepasa los 60 años. Esta mayoría femenina en el caso de los $\mathrm{CF}$, ha sido ampliamente documentada.19,20

El 56,6\% de los cuidadores y el $69 \%$ de las personas con EC residen en la zona urbana, con un nivel educativo medio-bajo, y la mitad aproximadamente cuenta con pareja estable y un trabajo remunerado, en el $46,9 \%$ de los casos.
La situación de EC y el cuidado requerido se presentan para el $67,4 \%$ de las personas con EC y el $72 \%$ de los cuidadores, hace más de un año. Varias de estas características son comunes a varios grupos de personas con EC - CF estudiadas.21 Para ellos, la familia es la principal fuente de apoyo social que tienen las personas cuando viven situaciones de enfermedad crónica.22

El 30,1\% de personas con EC y el $77 \%$ de los CF tienen un compromiso religioso medio o alto, siendo la mayor parte de ellos de religión católica. Este hallazgo refleja una vez más dos aspectos: el primero que frente a la condición de EC el componente religioso de la espiritualidad se fortalece, el segundo, que los CF tienden a verse más comprometidos en este ámbito de la vida con la experiencia.23 
El estado de salud de las personas con EC refleja que el 34\% tiene alteración funcional y el 21\%, alteración cognitiva. En ese sentido es preocupante pero no sorpresivo que el $8,6 \%$ de los CF también refieran limitación y el 39,7\% enfermedad, especialmente metabólica, lo que puede estar asociado con el desgaste generado por esta responsabilidad.24,25

La enfermedad cardiovascular, el cáncer, la enfermedad neurológica, la del aparato locomotor y la enfermedad renal reflejan una vez más el perfil epidemiológico del país.1

En el contexto colombiano las intervenciones de soporte social con TIC han demostrado buenos resultados.26 Uno de los mecanismos más innovadores para dar apoyo social en situaciones de EC son las TIC.27 Sin embargo, los hallazgos del presente estudio dejan ver que la apropiación de las TIC es heterogénea, con mayores niveles en el uso del radio y la televisión, para lo cual existen muy pocos desarrollos de apoyo en el cuidado.

La evidencia existente sobre estudios realizados en diferentes grupos poblacionales, en especial, en personas con EC, concluyen que, como en este caso, la familia es el principal proveedor de apoyo social y de apoyo emocional, a través del afecto y la afirmación; en el caso de la EC, éste apoyo permite asumir una actitud positiva ante la misma y ayuda a las personas a reducir el estrés en la vida.6

El soporte social aunque es un fenómeno complejo, se fundamenta en el planteamiento de que éste, puede proteger a las personas en crisis de una gran variedad de estados patológicos que incluyen la $\mathrm{EC}_{.6,28,29}$

En el caso de los CF ellos se encuentran satisfechos con el soporte social percibido, en especial en las regiones Amazonía y Orinoquía. Estudios anteriores que miraron la percepción de soporte social en diferentes regiones de Colombia, tienen distintos reportes. En el Caribe se encuentra que los CF están satisfechos con el apoyo social percibido, especialmente en las categorías: "interacción personal" y "educación e información" y que este es recibido de parte de los integrantes habituales de su red. ${ }_{30}$ En la Orinoquía se refiere una respuesta adaptativa positiva por parte de los CF y se señala que su bienestar redunda en el de sus familiares enfermos.31 En la región Andina por el contrario, el nivel de insatisfacción es mayor con un poco más de la mitad de los CF en esta condición, en todas las dimensiones estudiadas. 32

Ahora bien, respecto al apoyo emocional se considera fundamental para procesos de afrontamiento, estrés, y manejo de crisis de las personas con EC y los CF. Contar con personas representativas que puedan escuchar, aconsejar, apoyar en la obtención de información, que brinden confianza, respaldo y faciliten espacios para verbalizar temores y preocupaciones, son determinantes claves en la vivencia de la cronicidad. Se identifica una falencia importante de los participantes en contar con este tipo de apoyo emocional efectivo, situación que genera probablemente mayor estrés, y dificultades en el proceso de afrontamiento de la enfermedad.

Los resultados tienen similitud con lo encontrado en los trabajos de Ramírez et al.,33 Herrera et al.,30 Díaz,34 en lo referente a las categorías de la interacción social, tanto en los pacientes, como en los CF en aspectos que corresponden a las situaciones de distracción, recreación, los intereses en particular y el acompañamiento de la red de soporte social, como un factor que ayuda a disminuir la carga de cuidado.

Aunque de manera global, se reconoce una significativa red de apoyo informal para las personas con enfermedad crónica, constituida por sus amigos y parientes, de manera similar a la de los cuidadores familiares, hallazgos similares a lo descrito por Amaya y Carrillo.35 Así mismo, llama la atención un número importante de pacientes que están solos o cuentan con un único amigo/pariente para apoyarse en su cuidado. Por otro lado, cabe señalar que es paradójica la identificación que hacen, tanto los cuidadores, como los pacientes del número de parientes o amigos con los que cuenta, versus la efectividad de estos en cada uno de los componentes del apoyo social. 
Son limitaciones identificadas en el estudio, los posibles sesgos de selección al ser una muestra a conveniencia como primer referente de los centros participantes, el número de usuarios por región para lograr igual representación, y la dificultad de estudiar a la diada (persona con enfermedad crónica y su cuidador familiar), lo que impidió la comparación del soporte social percibido entre los dos actores.

Estos resultados son un primer referente para Colombia sobre soporte social percibido, tanto en personas con EC, como en los cuidadores familiares participantes de diferentes regiones del país, que aporta en la consolidación de una línea base para contar con un diagnóstico de las cinco macroregiones de Colombia. En futuros estudios es necesario abordar submuestras de pacientes y cuidadores que exploren la asociación de variables sociodemográficas, tipo y fase de la enfermedad crónica, escenario de captación (hospitalizados o consulta externa) y aspectos socioculturales propios de las regiones, que puedan incidir en la percepción del soporte social. Así mismo, se requiere diseñar y validar propuestas de intervención acordes con estas condiciones.

\section{Conclusiones}

Los hallazgos de este estudio reflejan que las personas con enfermedad crónica y sus cuidadores familiares tienen niveles de soporte social percibido, heterogéneos en las diferentes macro regiones geográficas de Colombia y que el mismo, se aleja de manera importante de ser el óptimo. Se evidencia que existen grupos de apoyo informal constituidos por parientes y amigos, sin embargo, las diferentes formas en que estos brindan el soporte social no son efectivas, ni parecen estar acordes con las demandas de cuidado. En tal sentido, el soporte social percibido por la diada receptor - cuidador familiar, debe abordarse a partir de las redes de apoyo informal y formal, incluyendo a la familia, las comunidades, las organizaciones de usuarios, las instituciones y el mismo Sistema General de Seguridad Social en Salud, para buscar modificar de forma efectiva una realidad compleja y de alto impacto en la salud del país.

Es preciso señalar que en Colombia, como en la mayor parte de los países de América Latina, la inequidad es alta y a pesar de los avances, aún se expresa en los sistemas de salud.36 Estas diferencias se reflejan en muchos de los aspectos de la vida cotidiana, como se ilustra en este estudio frente a las características y percepción de soporte social en la vida y el cuidado de personas con enfermedad crónica.

Financiación: Programa para la Disminución de la Carga de la Enfermedad Crónica no Trasmisible en Colombia, con el apoyo financiero de COLCIENCIAS, código 501753730890.

Conflicto de intereses: Ninguno declarado por los autores.

\section{Referencias}

1. Organización Mundial de la Salud. Estadísticas sanitarias mundiales 2014.

2. República de Colombia. Ministerio de Salud. Guía de instrucción para el diseño de programas de gestión de enfermedades crónicas. 2014.

3. Sayers SL, Riegel B, Pawlowski S, Coyne JC, Samaha FF. Social support and self-care of patients with heart failure. Annals of Behavioral Medicine. 2008; 35(1):709.

4. Gómez MM, Lagoueyte MI. El apoyo social: Estrategia para aforntar el cáncer de cérvix. Avances en Enfermería. 2012;30(1):32-41.

5. Senuzun A, Bilge A, Mermer G, Sahin S. Validity and reliability of the social support scale in chronic diseases-TR (SSCII-TR). European Geriatric Medicine. 2014; 5: 21-25.

6. Vega-Angarita O, González-Escobar D. Apoyo social: elemento clave en el afrontamiento de la enfermedad crónica. Enfermería Global. 2009(16):0-.

7. Durá E, Garcés J. La teoría del apoyo social y sus implicaciones para el ajuste psicosocial de los enfermos oncológicos. Revista de Psicología Social. 1991; 6(2):257-71.

8. Romero E, Herrera A, Florez I, Montalvo A. Las TIC y el apoyo social: una respuesta desde enfermería en Cartagena (Colombia). Salud Uninorte. 2010; 26(2):325-38.

9. Carrillo G, Sánchez B, Barrera L. Habilidad de cuidado de cuidadores familiares de niños y adultos con enfermedad crónica. Index de enfermería: información bibliográfica, investigación y humanidades. 2014; 23(3):129-133. 
10. Sorkin D, Rook KS, Lu JL. Loneliness, lack of emotional support, lack of companionship, and the likelihood of having a heart condition in an elderly sample. Annals of Behavioral Medicine. 2002; 24(4):290-8.

11. Krumholz HM, Butler J, Miller J, Vaccarino V, Williams CS, de Leon CFM, et al. Prognostic importance of emotional support for elderly patients hospitalized with heart failure. Circulation. 1998; 97(10):958-64.

12. Berkman LF, Leo-Summers L, Horwitz RI. Emotional support and survival after myocardial infarction: a prospective, population-based study of the elderly. Annals of Internal Medicine. 1992; 117(12):1003-9.

13. Godoy AM, Pérez MA, Doménech del Rio A. Prunera MJ. Caregiver burden and social support perceived bu patients with chronic obstructive pulmonar disease. Rev Calid Asist. 2014; 29(6):320-324.

14. República de Colombia. Ministerio de Salud. Resolución 8430 de 1993 (octubre 4): Por la cual se establecen las normas científicas, técnicas y administrativas para la investigación en salud. Bogotá, D.C.; octubre 4 de 1993.

15. República de Colombia. Universidad Nacional de Colombia, Consejo Superior Universitario. Acuerdo 016 de 2011 (octubre 18): Por el cual se establece la Política Ambiental de la Universidad Nacional de Colombia. Bogotá, D.C.: Sistema de Información Normativa, Jurisprudencial y de Conceptos “Régimen Legal”. 2011.

16. Sherbourne C, Stewart A. Themos social support survey. Soc Sci Med. 1991;32(6):705-14.

17. Rodríguez-Espínola S, Enrique HC. Validación Argentina del cuestionario MOS de apoyo social percibido. Psicología, Cultura y Sociedad. 2006.

18. Londoño N, Rogers KM, Castilla J, Posada S, Ochoa N, Jaramillo $M$, et al. Validación en Colombia del cuestionario MOS de apoyo social. International Journal of Psychological Research. 2012;5(1):142-50.

19. Bover A, Gastaldo D. La centralidad de la familia como recurso en el cuidado domiciliario: perspectiva de género y generación. Rev Bras Enferm. 2005; 58(1):916.

20. Vaquiro-Rodríguez S, Stiepovich Bertoni J. Cuidado informal, un reto asumido por la mujer. Ciencia y enfermería. 2010;16(2):17-24.

21. Espín-Andrade AM. Caracterización psicosocial de cuidadores informales de adultos mayores con demencia. Revista Cubana de Salud Pública. 2008; 34(3):0-

22. Ledón L. Enfermedades crónicas y vida cotidiana. Revista Cubana de Salud Pública. 2011;37(4):488-99.

23. Sánchez B. Dimensión espiritual del cuidado de enfermería en situaciones de cronicidad y muerte. Aquichan. 2004;4(4):6-9.

24. Giraldo CI, Franco GM. Calidad de vida de los cuidadores familiares. Aquichan. 2006;6(1):38-53.
25. Zambrano-Cruz R, Ceballos-Cardona P. Síndrome de carga del cuidador. Revista Colombiana de Psiquiatría. 2007;36:26-39.

26. Vega OM, González DS. Soporte social mediante intervención telefónica en cuidadores de enfermos crónicos discapacitados en Cúcuta, Colombia. Revista Respuestas. 2013;18(1):32-42.

27. Cárdenas-Corredor DC, Melenge-Díaz B, Pinilla J, Carrillo-González GM, Chaparro-Díaz L. Soporte social con el uso de las TIC para cuidadores de personas con enfermedad crónica: un estado del arte. Aquichan. 2010;10(3):204-213.

28. Harley C, Pini S, Bartlett YK, Velikova G. Defining chronic cancer: patient experiences and selmanagement needs. BMJ Support Palliative Care. 2015;5(4):343-350.

29. Hernández NE, Moreno CM, Barragán JA. Necesidades de cuidado de la díada cuidador-persona: expectativa de cambio en intervenciones de enfermería. Revista Cuidarte. 2014;5(2):748-56.

30. Herrera A, Montalvo A, Flórez I, Romero E. Soporte social a cuidadores familiares de personas con enfermedad crónica en Cartagena. Aquichan. 2012; 12(3):286-297.

31. Pinzón M, Aponte L, Galvis C. Perfil de los cuidadores informales de personas con enfermedades crónicas y calidad de vida, Villavicencio. Meta. Orinoquia 2012;16(2):107-17.

32. Vega OM. Percepción del apoyo social funcional en cuidadores familiares de enfermos crónicos. Aquichan. 2011;11(3):274-86.

33. Ramírez MM, Ramírez A, Vergel L, Santafé L. Línea de atención para el cuidador principal de pacientes oncológicos con soporte social. Revista Ciencia y Cuidado. 2012;9(1):34-42.

34. Díaz L. Relación entre el soporte social percibido y la habilidad de cuidar de los cuidadores principales de niños que viven en situación de enfermedad crónica. Tesis de Maestría. Bogotá: Universidad Nacional de Colombia Facultad de Enfermería. 2003.

35. Amaya MC, Carrillo GM. Apoyo social percibido y afrontamiento en personas con dolor crónico no maligno. Aquichan. 2015;15(4):461-474.

36. Calderón CAA, Botero JC, Bolaños JO, Martínez RR. Sistema de salud en Colombia: 20 años de logros y problemas. The colombian healthcare system: 20 years of achievements and problems. Ciênc saúde coletiva. 2011;16(6):2817-28. 